Research Article

\title{
Free-Interface Modal Synthesis Based Substructural Damage Detection Method
}

\author{
Shanghong Chen, Wei Lin, Jiexin Yu, and Ai Qi \\ School of Civil Engineering, Fuzhou University, Fuzhou 350116, China \\ Correspondence should be addressed to Wei Lin; cewlin@fzu.edu.cn \\ Received 17 April 2014; Accepted 17 June 2014; Published 15 July 2014 \\ Academic Editor: Bo Chen
}

Copyright (C) 2014 Shanghong Chen et al. This is an open access article distributed under the Creative Commons Attribution License, which permits unrestricted use, distribution, and reproduction in any medium, provided the original work is properly cited.

Free-interface modal synthesis method is applied to civil structure, and a substructure method is proposed by introducing the method into global sensitivity method. The substructure expression of the derivatives of eigenvalues and eigenvectors with respect to elemental parameters is obtained. The accuracy of the application of free-interface modal synthesis method is evaluated with different retained modes in substructure, and then the effectiveness of the proposed substructure sensitivity method is illustrated through an 11-storey building under both single- and multidamage cases. Both the damage locations and the extent can be effectively identified. By comparing it with the identical results of global sensitivity method, the proposed method can be faster in detecting the damage location and more stable under multidamage cases. Since this substructure sensitivity method only needs to update sensitivity matrix in the substructure with relative small number of DOFs, it may save much computation effort and become more efficient.

\section{Introduction}

To ensure the safety of large-scale civil infrastructures, more and more health monitoring systems are adopted to evaluate health condition and also to prevent sudden failure due to accumulation of component damages [1-5]. Nowadays, although more sensors were installed and varieties of data were collected, it encounters a problem on how to derive useful information or identify some early damage through the massive data [6,7]. An accurate finite element model (FEM) is usually required to provide a baseline of the healthy structure, but, due to uncertainties from geometry, material properties, and boundary conditions, the FEM needs to be first updated through the picked up information $[8,9]$. Moreover, by comparing the output change such as natural frequency, mode shape, mode shape curvature, and flexibility changes due to structural aging or adverse environmental effects, it is expected that the system should be able to detect local damages and give maintenance advices [10-12]. As it comes to civil structures, their FEMs usually have large number of DOFs, which will result in equations with huge dimension during the model updating and damage detection procedure. And it would take on a lot of resources and make the calculation to be very time-consuming [13-15].

Sensitivity-based method is believed to be an effective way in model updating and damage detection. It is based on the fact that a perturbation in parameters will affect the outputs of a structure, such as frequencies and mode shapes [16]. And the derivative of target function with respect to system parameters is defined as sensitivity. Many efforts have been made by researchers [17-21]. In these sensitivity methods, a sensitivity matrix has to be computed at each time step. It will be a tedious work if too many uncertainties are assumed. Substructure method [22] may be an effective approach to the problem. The main idea is to divide a whole structure into many substructures first, and then the calculation can be achieved independently in each substructure. Finally, reconstruction work is done based on the boundary conditions. Chen and Bicanic [20] first proposed a substructure method to calculate eigenvalue and eigenvector. Kron and Li et al. [23-27] proposed a substructure method in time domain to identify local damage under moving vehicular loads without knowledge of the vehicle properties and the time-histories of moving interaction forces. And Weng et al. [28, 29] extent 
Kron's method and proposed an inverse substructure method in frequency domain.

This paper attempted to apply free-interface modal synthesis method in civil infrastructure and to introduce the method into the traditional global sensitivity method and to establish a substructure sensitivity method. The derivatives of eigenvalue and eigenvector with respect to system parameters will be calculated with the substructure method. With only those factors within certain substructures having nonzero value, this method is supposed to require few computation efforts and higher efficiency in damage detection. Numerical example is conducted on an 11-storey frame building. In order to maintain the accuracy during damage detection, the accuracy of modal synthesis method will be first discussed and then the damage detection ability of the proposed method will be verified under several damage cases.

\section{Free-Interface Modal Synthesis}

The idea of modal synthesis method is to first divide a structure into a series of substructures with no bound constraints attached. After acquiring the dynamic characteristics of every substructure, the system equation can be reconstructed by taking the displacement coordinate of each substructure into account, and finally the modal information can be derived from this equation. To facilitate the description, it is assumed that the structure is divided into only two substructures, named substructures $a$ and $b$. And modal synthesis method with more substructures concerned can also be derived from the following equations.

2.1. Basic Modal Equation of a Substructure. Similar to the whole structure, taking substructure $a$ as an example, the equations of motion with free vibration case can be expressed as

$$
\left[{ }^{(a)} M\right]\left\{{ }^{(a)} \ddot{x}\right\}+\left[{ }^{(a)} K\right]\left\{{ }^{(a)} x\right\}=\left\{{ }^{(a)} f\right\},
$$

where $\left[{ }^{(a)} M\right]$ and $\left[{ }^{(a)} K\right]$ are the mass and stiffness matrices of substructure $a,\left\{{ }^{(a)} \ddot{x}\right\}$ and $\left\{{ }^{(a)} x\right\}$ represent the substructure's acceleration and displacement vectors in normal coordinate, respectively, and $\left\{{ }^{(a)} f\right\}$ is the applied forces on the corresponding DOFs of substructure $a$.

The eigenvalues and eigenvectors can be solved from the corresponding modal equation in frequency domain. And if only the first $q$ modes are considered and compose a matrix $\left[{ }^{(a)} \Phi\right]$, then the displacement vector $\left\{{ }^{(a)} x\right\}$ can be decomposed as the following equation which is called the first coordinate transformation:

$$
\left\{{ }^{(a)} x\right\}=\left[{ }^{(a)} \Phi\right]\left\{{ }^{(a)} p\right\}
$$

where $\left\{{ }^{(a)} p\right\}$ represents the responses in modal coordinate.

The nodes of the substructure can be divided into two sets: those attached to the interface and those with only internal forces applied on. It is expressed as subscription $I$ and $J$, respectively. Then, the displacement vector can be expressed as

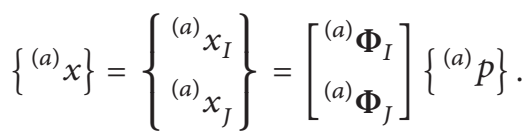

By normalizing using mass matrix, the dominate equation of $\left\{{ }^{(a)} p\right\}$ in modal coordinate is

$$
\left[{ }^{(a)} \widetilde{M}\right]\left\{{ }^{(a)} \ddot{p}\right\}+\left[{ }^{(a)} \widetilde{K}\right]\left\{{ }^{(a)} p\right\}=\left[{ }^{(a)} \Phi\right]\left\{{ }^{(a)} f\right\}
$$

where $\left[{ }^{(a)} \widetilde{M}\right]$ and $\left[{ }^{(a)} \widetilde{K}\right]$ are the normalized mass and stiffness matrix of substructure $a$, and the following modal equation can be further derived:

$$
\left\{{ }^{(a)} \ddot{p}\right\}+\left[{ }^{(a)} \Omega\right]\left\{{ }^{(a)} p\right\}=\left[{ }^{(a)} \Phi\right]\left\{{ }^{(a)} f\right\}
$$

in which $\left[{ }^{(a)} \Omega\right]=\operatorname{diag}\left({ }^{(a)} \lambda_{1},{ }^{(a)} \lambda_{2}, \ldots,{ }^{(a)} \lambda_{q}\right)$ is a matrix containing the eigenvalues of selected substructure and, in accordance with diagonal arrangement, $\left\{{ }^{(a)} f\right\}$ is the interface force vector.

2.2. Interface Coordination Conditions. Assuming that only the first $n$ and $m$ modes of substructures $a$ and $b$ are considered during modal synthesis and the number of interface DOFs of the substructure is $J$, since the two substructures share the same nodes at the interface, there should be displacement coordination equations as

$$
\left\{{ }^{(a)} x_{J}\right\}=\left\{{ }^{(b)} x_{J}\right\}
$$

By using (2), this equation can also be written in modal coordinate as

$$
\left[{ }^{(a)} \boldsymbol{\Phi}_{J}\right]\left\{{ }^{(a)} p\right\}=\left[{ }^{(b)} \boldsymbol{\Phi}_{J}\right]\left\{{ }^{(b)} p\right\}
$$

In order to reconstruct the system equation, it is supposed that the number of the retained modes of each substructure should exceed the number of DOFs of the interface $J$, that is, to require $n>J$ and $m>J$, and $\left[{ }^{(a)} \Phi_{J}\right]$ can be written as

$$
\left[{ }^{(a)} \boldsymbol{\Phi}_{J}\right]=\left[{ }^{(a)} \boldsymbol{\Phi}_{J S}:{ }^{(a)} \boldsymbol{\Phi}_{J R}\right]
$$

where $\left[{ }^{(a)} \boldsymbol{\Phi}_{J S}\right.$ ] is a nonsingular matrix derived from [ $\left[{ }^{(a)} \boldsymbol{\Phi}_{J}\right]$ and the rest modal vector are formed as $\left[{ }^{(a)} \boldsymbol{\Phi}_{J R}\right]$. Substituting (8) into (7) yields

$$
\left[{ }^{(a)} \boldsymbol{\Phi}_{J S}:{ }^{(a)} \boldsymbol{\Phi}_{J R}\right]\left\{\begin{array}{c}
{ }^{(a)} p_{J S} \\
{ }^{(a)} p_{J R}
\end{array}\right\}=\left[{ }^{(b)} \boldsymbol{\Phi}_{J}\right]\left\{{ }^{(b)} p\right\}
$$


It can be noticed from (9) that those independent modal terms $\left\{{ }^{(a)} p_{J S}\right\}$ can be expressed as

$$
\begin{gathered}
\left\{{ }^{(a)} p_{J S}\right\}=-\left[{ }^{(a)} \boldsymbol{\Phi}_{J S}\right]^{-1}\left[{ }^{(a)} \boldsymbol{\Phi}_{J R}\right]\left\{{ }^{(a)} p_{J R}\right\} \\
+\left[{ }^{(a)} \boldsymbol{\Phi}_{J S}\right]^{-1}\left[{ }^{(b)} \boldsymbol{\Phi}_{J}\right]\left\{{ }^{(b)} p\right\} \\
\{p\}=\left\{\begin{array}{c}
{ }^{(a)} p \\
{ }^{(b)} p
\end{array}\right\}=\left\{\begin{array}{c}
{ }^{(a)} p_{J S} \\
{ }^{(a)} p_{J R} \\
{ }^{(b)} p
\end{array}\right\}=[T]\{q\},
\end{gathered}
$$

in which

$$
\begin{gathered}
{[T]=\left(\begin{array}{cc}
-\left[{ }^{(a)} \boldsymbol{\Phi}_{J S}\right]^{-1}\left[{ }^{(a)} \boldsymbol{\Phi}_{J R}\right]\left[{ }^{(a)} \boldsymbol{\Phi}_{J S}\right]^{-1}\left[{ }^{(b)} \boldsymbol{\Phi}_{J}\right] \\
{[I]} & 0 \\
0 & {[I]}
\end{array}\right)} \\
\{q\}=\left\{\begin{array}{c}
\left({ }^{(a)} p_{J R}\right. \\
{ }^{(b)} p
\end{array}\right\} .
\end{gathered}
$$

Matrix $[T]$ here can be considered as a transformation matrix for the second coordinate transformation, and $\{q\}$ is the generalized coordinate of the remaining DOFs in this coordinate.

2.3. Reconstructed Modal Equation. Since the boundary condition is assumed to be free for substructures $a$ and $b$, the following modal equation can be obtained by simply putting their modal equations together:

$$
\begin{gathered}
\left\{\begin{array}{c}
{ }^{(a)} \ddot{p} \\
{ }^{(b)} \ddot{p}_{b}
\end{array}\right\}+\left(\begin{array}{cc}
{\left[{ }^{(a)} \Omega\right]} & 0 \\
0 & {\left[{ }^{(b)} \Omega\right]}
\end{array}\right)\left\{\begin{array}{l}
{ }^{(a)} p \\
{ }^{(b)} p
\end{array}\right\} \\
=\left(\begin{array}{cc}
{\left[{ }^{(a)} \boldsymbol{\Phi}_{J}\right]^{T}} & 0 \\
0 & {\left[{ }^{(b)} \boldsymbol{\Phi}_{J}\right]^{T}}
\end{array}\right)\left\{\begin{array}{c}
{ }^{(a)} f_{J} \\
{ }^{(b)} f_{J}
\end{array}\right\} .
\end{gathered}
$$

By substituting the second coordinate transformation equation (11) and multiplying $[T]^{T}$ in both ends plus considering that the reaction forces are coupled in the interface nodes, one can reconstruct the system modal equation as

$$
\begin{gathered}
{\left[M^{*}\right]\{\ddot{q}\}+\left[K^{*}\right]\{q\}} \\
=[T]^{T}\left(\begin{array}{cc}
{\left[{ }^{(a)} \boldsymbol{\Phi}_{J}\right]^{T}} & 0 \\
0 & {\left[{ }^{(b)} \boldsymbol{\Phi}_{J}\right]^{T}}
\end{array}\right)\left\{\begin{array}{l}
{ }^{(a)} f_{J} \\
{ }^{(b)} f_{J}
\end{array}\right\}=0 \\
\left\{{ }^{(a)} f_{J}\right\}+\left\{{ }^{(b)} f_{J}\right\}=0,
\end{gathered}
$$

where

$$
\left[M^{*}\right]=[T]^{T}[T] \quad\left[K^{*}\right]=[T]^{T} \operatorname{diag}\left({ }^{(a)} \Omega,{ }^{(b)} \Omega\right)[T] .
$$

By solving (16), the expression of eigenvalue and eigenvector in this coordinate can be obtained, by further using
(2) and (11) for inverse coordinate transformation and then comes the eigenvalue and eigenvector of the whole structure:

$$
\{x\}=\left[\begin{array}{lll}
{\left[{ }^{(a)} \boldsymbol{\Phi}\right]} & \\
& & {\left[{ }^{(b)} \boldsymbol{\Phi}\right]}
\end{array}\right][T][q] .
$$

\section{Modal Synthesis Based Substructure Damage Identification}

3.1. Sensitivity Analysis. The dynamic response sensitivitybased model updating method is adopted here with

$$
R_{e}-R_{0}=S\left\{r_{u}-r_{o}\right\} \Longleftrightarrow \Delta R=\left.S\right|_{m \times n} \Delta r,
$$

where $\Delta r$ is the perturbation of the vector of structural elemental stiffness factors and $\Delta R$ is the changed structural responses due to damage. And $S$ is the sensitivity matrix of the structural responses with respect to the stiffness factors.

Equation (18) implies that, when performing sensitivity analysis, the sensitivity matrix of the responses with respect to each elemental stiffness factor should be calculated on each time step. For structures with large DOFs, the calculation burden will be huge. Therefore, instead of calculating the sensitivity matrix in the whole structure, it is expected by introducing the substructure method; the calculation can be done in parallel in different substructure. Moreover, for civil structures, usually there are key parts or components which people may concern most. In such situation, one can assume that damage only occurs in certain substructures and there are non-zero values only for those corresponding terms in sensitivity matrix.

3.2. Derivatives of Eigenvalue with Substructure Method. The basic modal equation expressed as equation (14) is written as a truncated Taylor series

$$
\frac{\partial\left(K^{*}-M^{*} \lambda_{i}\right)}{\partial r}\left\{q_{i}\right\}+\left(K^{*}-M^{*} \lambda_{i}\right) \frac{\partial\left\{q_{i}\right\}}{\partial r}=0,
$$

where the parameter $r$ here still represents the changing factor of the whole structure, by multiply $\{q\}^{T}$ on both ends achieves

$$
\left\{q_{i}\right\}^{T} \frac{\partial\left(K^{*}-M^{*} \lambda_{i}\right)}{\partial r}\left\{q_{i}\right\}+\left\{q_{i}\right\}^{T}\left(K^{*}-M^{*} \lambda_{i}\right) \frac{\partial\left\{q_{i}\right\}}{\partial r}=0 .
$$

And because of the symmetry of $\left(K^{*}-M^{*} \lambda_{i}\right)$ and noticing $\left(K^{*}-M^{*} \lambda_{i}\right)\left\{q_{i}\right\}=0$, the above equation can be simplified as

$$
\left\{q_{i}\right\}^{T} \frac{\partial\left(K^{*}-M^{*} \lambda_{i}\right)}{\partial r}\left\{q_{i}\right\}=0 .
$$

Then, with substitution of $\left[M^{*}\right]$ and $\left[K^{*}\right]$ into (21), it can be transformed to

$$
\left\{q_{i}\right\}^{T} T^{T} \frac{\partial\left(\Omega_{m}{ }^{p}-\lambda_{i}\right)}{\partial r} T\left\{q_{i}\right\}=0 .
$$


Denoting $\left\{p_{i}\right\}=T\left\{q_{i}\right\}$, the above equation can be rewritten as

$$
\left\{p_{i}\right\}^{T} \frac{\partial\left(\Omega_{m}^{p}-\lambda_{i}\right)}{\partial r}\left\{p_{i}\right\}=0 .
$$

And the first-order derivative of the eigenvalue with respect to elemental parameter can be expressed as

$$
\frac{\partial \lambda_{i}}{\partial r}=\left\{p_{i}\right\}^{T} \frac{\partial \Omega_{m}^{p}}{\partial r}\left\{p_{i}\right\} .
$$

Since each substructure is independent, the derivative can be calculated solely in certain substructure which contains the element parameters; otherwise, the irrelevant terms should return a zero value. Consider

$$
\begin{gathered}
\frac{\partial \Omega_{m}{ }^{p}}{\partial r}=\left(\begin{array}{ccc}
0 & 0 & 0 \\
0 & \frac{\partial \Omega_{m}{ }^{(r)}}{\partial r} & 0 \\
0 & 0 & 0
\end{array}\right), \\
\frac{\partial \Phi_{m}^{p}}{\partial r}=\left(\begin{array}{ccc}
0 & 0 & 0 \\
0 & \frac{\partial \Phi_{m}{ }^{(r)}}{\partial r} & 0 \\
0 & 0 & 0
\end{array}\right),
\end{gathered}
$$

in which $\Omega_{m}{ }^{p}$ can be calculated by first normalizing the substructures' mass and stiffness matrix and then putting them into one diagonal matrix:

$$
\begin{array}{cc}
{\left[\boldsymbol{\Phi}^{a}\right]^{T} M^{a}\left[\boldsymbol{\Phi}^{a}\right]=I_{N^{a}},} & {\left[\boldsymbol{\Phi}^{a}\right]^{T} K^{a}\left[\boldsymbol{\Phi}^{a}\right]=\Omega^{a}} \\
{\left[\boldsymbol{\Phi}^{b}\right]^{T} M^{b}\left[\boldsymbol{\Phi}^{b}\right]=I_{N^{b}},} & {\left[\boldsymbol{\Phi}^{b}\right]^{T} K^{b}\left[\boldsymbol{\Phi}^{b}\right]=\Omega^{b}} \\
\Omega^{p}=\left(\begin{array}{cc}
\Omega^{a} & 0 \\
0 & \Omega^{b}
\end{array}\right), & \boldsymbol{\Phi}^{p}=\left(\begin{array}{cc}
\boldsymbol{\Phi}^{a} & 0 \\
0 & \boldsymbol{\Phi}^{b}
\end{array}\right) .
\end{array}
$$

By further applying modal truncation and sparse matrix storage in the above procedure, the system resource will be greatly saved during the construction of sensitivity matrix. Subscription $m$ and $r$ refer to the "master" and "residual," respectively. By putting the master or residual eigenvectors of the substructures together, one can get

$$
\begin{array}{rlr}
\Omega_{m}{ }^{p}=\left(\begin{array}{cc}
\Omega_{m}{ }^{a} & 0 \\
0 & \Omega_{m}{ }^{b}
\end{array}\right), & \Omega_{s}{ }^{p}=\left(\begin{array}{cc}
\Omega_{s}{ }^{a} & 0 \\
0 & \Omega_{s}{ }^{b}
\end{array}\right) \\
\boldsymbol{\Phi}_{m}{ }^{p}=\left(\begin{array}{cc}
\boldsymbol{\Phi}_{m}{ }^{a} & 0 \\
0 & \boldsymbol{\Phi}_{m}{ }^{b}
\end{array}\right), & \boldsymbol{\Phi}_{s}{ }^{p}=\left(\begin{array}{cc}
\boldsymbol{\Phi}_{s}{ }^{a} & 0 \\
0 & \boldsymbol{\Phi}_{s}^{b}
\end{array}\right) .
\end{array}
$$

3.3. Derivatives of Eigenvector with Substructure Method. By using free-interface modal synthesis method mentioned above, the $i$ th eigenvector can be expressed as

$$
\bar{\phi}_{i}=\boldsymbol{\Phi}_{m}^{p}\left\{p_{i}\right\}=\boldsymbol{\Phi}_{m}^{p} T\left\{q_{i}\right\} .
$$

Differentiating this equation with respect to some elemental parameter $r$ results in

$$
\frac{\partial \bar{\phi}_{i}}{\partial r}=\frac{\partial \boldsymbol{\Phi}_{m}^{p}}{\partial r} T\left\{q_{i}\right\}+\boldsymbol{\Phi}_{m}^{p} T\left\{\frac{\partial q_{i}}{\partial r}\right\},
$$

where $\partial \Phi_{m}{ }^{p} / \partial r$ represents the derivative of master modes' eigenvector with respect to parameter $r$. It is noticed that the equation can be solved once $\left\{\partial q_{i} / \partial r\right\}$ was obtained.

Assume that

$$
\left\{\frac{\partial q_{i}}{\partial r}\right\}=\left\{v_{i}\right\}+c_{i}\left\{q_{i}\right\}
$$

For $\left\{v_{i}\right\}$, substituting (29) into (19) gives

$$
\left(K^{*}-M^{*} \lambda_{i}\right)\left(\left\{v_{i}\right\}+c_{i}\left\{q_{i}\right\}\right)=-\frac{\partial\left(K^{*}-M^{*} \lambda_{i}\right)}{\partial r}\left\{q_{i}\right\} .
$$

Noticing that $\left(K^{*}-M^{*} \lambda_{i}\right)\left\{q_{i}\right\}=0$ and substituting (16) into the equation, (30) can be simplified as

$$
X_{i}\left\{v_{i}\right\}=\left\{Y_{i}\right\},
$$

where

$$
\begin{gathered}
X_{i}=\left(K^{*}-M^{*} \lambda_{i}\right) \\
\left\{Y_{i}\right\}=-T^{T} \frac{\partial\left(\Omega_{m}{ }^{p}-\lambda_{i}\right)}{\partial r}[T]\left\{q_{i}\right\}=-T^{T} \frac{\partial\left(\Omega_{m}{ }^{p}-\lambda_{i}\right)}{\partial r}\left\{p_{i}\right\} .
\end{gathered}
$$

Considering that there are no duplicated frequencies, then the dimension of $X_{i}$ is $N_{m}{ }^{p}$ and the rank of this matrix should be $N_{m}{ }^{p}-1$. Denoting $k$ th term to be the largest term in $\left\{q_{i}\right\}$, by setting sum of the $k$ th row and column into zero, as well as $Y_{k}=0$, (31) becomes

$$
\left[\begin{array}{ccc}
X_{11} & 0 & X_{13} \\
0 & 1 & 0 \\
X_{31} & 0 & X_{33}
\end{array}\right]\left\{\begin{array}{l}
v_{i 1} \\
v_{i k} \\
v_{i 3}
\end{array}\right\}=\left\{\begin{array}{c}
Y_{i 1} \\
0 \\
Y_{i 3}
\end{array}\right\}
$$

from which $\left\{v_{i}\right\}$ can be solved.

In order to solve $c_{i}$, the orthogonally of the eigenvector can be expressed as

$$
\left\{p_{i}\right\}^{T}\left\{p_{i}\right\}=1 \quad\left\{q_{i}\right\}^{T} T^{T} T\left\{q_{i}\right\}=1 .
$$

Differentiating (34) with respect to $r$ gives

$$
\frac{\partial\left\{q_{i}\right\}^{T}}{\partial r} T^{T} T\left\{q_{i}\right\}+\left\{q_{i}\right\}^{T} T^{T} T \frac{\partial\left\{q_{i}\right\}}{\partial r}=0 .
$$

Then, substitute (29) into the above equation,

$$
\left(\left\{v_{i}\right\}^{T}+c_{i}\left\{q_{i}\right\}^{T}\right) T^{T} T\left\{q_{i}\right\}+\left\{q_{i}\right\}^{T} T^{T} T\left(\left\{v_{i}\right\}+c_{i}\left\{q_{i}\right\}\right)=0 .
$$

And the factor $c_{i}$ can be expressed as

$$
c_{i}=-\frac{1}{2}\left(\left\{v_{i}\right\}^{T} T^{T}\left\{p_{i}\right\}+\left\{p_{i}\right\}^{T} T\left\{v_{i}\right\}\right) .
$$

Thus, the first-order derivative of $\left\{q_{i}\right\}$ with respect to $r$ can be achieved as

$$
\left\{\frac{\partial q_{i}}{\partial r}\right\}=\left\{v_{i}\right\}-\frac{1}{2}\left(\left\{v_{i}\right\}^{T} T^{T}\left\{p_{i}\right\}+\left\{p_{i}\right\}^{T} T\left\{v_{i}\right\}\right)\left\{q_{i}\right\} .
$$

Finally, the derivative of eigenvector with respect to elemental parameter $r$ can be obtained through (29). 
3.4. Iterative Damage Detection Procedure. Initially, it is assumed that the analytical finite element model is intact, while damage is simulated as a decreasing of elastic modulus in certain element. The damage detection iterative procedure aims to detect the damage location and extent through the convergence of eigenvalue and eigenvector.

Step 1. Acquire the dynamic responses of damaged structure and transform these responses into frequency domain.

Step 2. Set the target function which is to be minimized. Usually, the target function vector will be formed by the first few eigenvalues and eigenvector listed together, and then the root-mean-square (RMS) value of the vector is the target function to be minimized.

Step 3. Construct the sensitivity matrix through substructure modal synthesis approach. The derivative of the target function with respect to every elemental stiffness factor should be calculated. The ones with eigenvalue and eigenvector terms can be solved with (24) and (39), respectively. The feature of the proposed substructural approach is that when calculating the sensitivity matrix, only those substructures which contain the elemental parameter will have non-zero value and need to be calculated; that is, the calculation will be limited to a much reduced dimension.

Step 4. Solve the perturbation vector of elemental stiffness factors $\Delta r$ from (18) with Least Squares Method or Tikhonov Regularization Technique.

Step 5. The elemental stiffness factors are iteratively updated with $r_{i+1}=r_{i}+\Delta r$ for the next iteration. Repeat Steps 2-5 until the convergence criterion is met to some level.

Step 6. Calculate the damage index which defines the damage extent of each element.

$$
\mathrm{SRF}_{i}=\frac{\Delta E_{i}}{E_{i}}=\frac{E_{i}^{u}-E_{i}^{o}}{E_{i}^{u}} .
$$

\section{Numerical Studies}

4.1. Modal Synthesis on a Frame Structure. Due to the mode truncation, there will be errors while using the modal synthesis method mentioned above. Since it is not expected too much errors caused by the uncertainty of the system parameters, the accuracy of the modal synthesis method is first examined by a simple example. As shown in Figure 1, numerical studies on an 11-storey frame structure are performed to examine the relationship between the number of maintained modes and the accuracy of this method. The finite element model of the structure consists of 24 nodes and 33 planar elements. It is considered that each node has 3 DOFs and there are a total number of 66 DOFs for the whole structure. The first 8 natural frequencies are calculated as $1.0353,3.3614,4.3592,6.3489,7.0573,10.147,11.536$, and $14.253 \mathrm{~Hz}$. The frame is disassembled into two substructures with 6 DOFs on the interface.

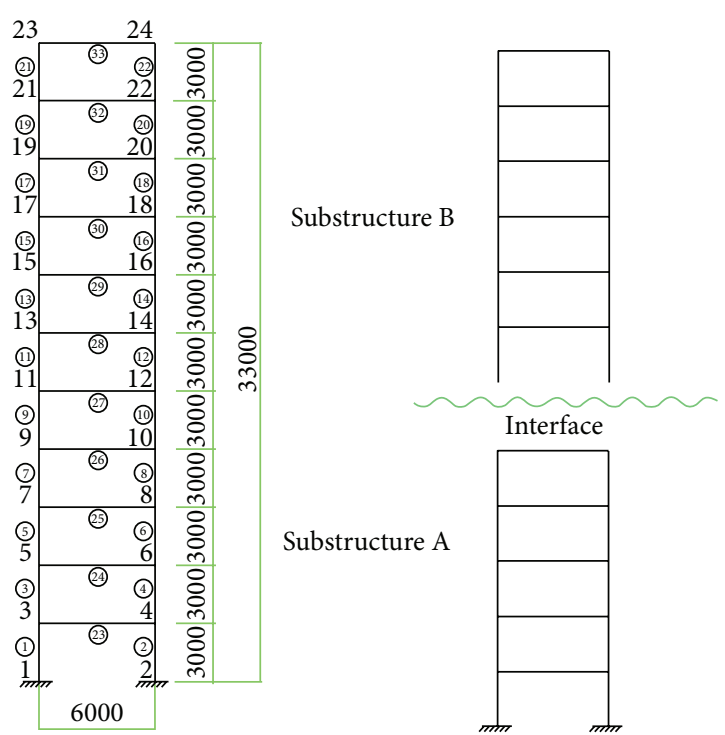

FIGURE 1: Eleven-storey frame structure (unit: mm).

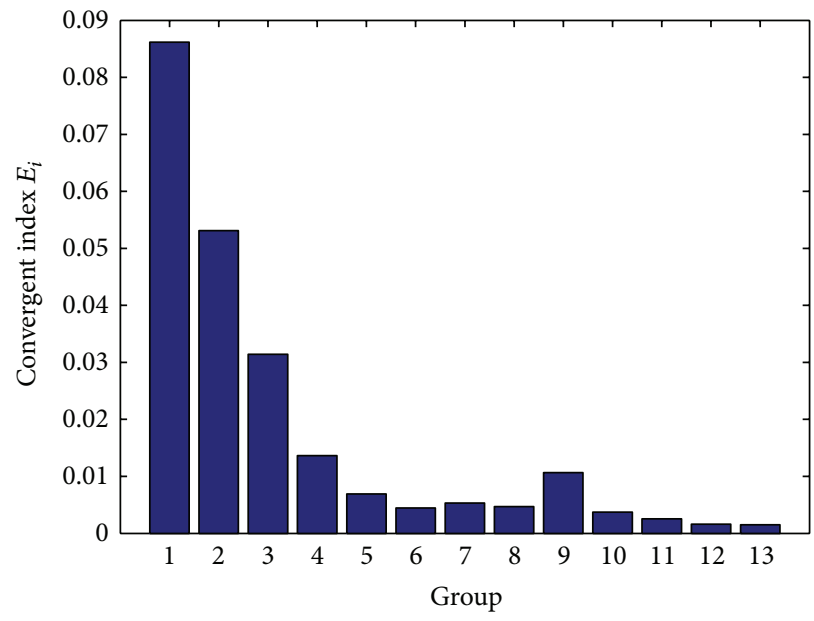

Figure 2: Convergent index $E_{i}$ with increasing retained modes.

The retained modes of the two substructures begin with 6 and 12 in Group 1 and increase by 2 for the next calculation until all the modes are included. Based on the modal analysis, it is expected that there should be a convergent point for the desired accuracy along with the increasing retained modes of the substructures. In this example, the first 8 eigenvalues and eigenvectors are selected to be examined. In order to further discuss how many modes should be retained, the following convergence index $E_{i}$ is defined. By gradually increasing the retained modes of the substructure, the sum of eigenvalues also increased, and here convergence index $E_{i}$ is calculated 
TABLE 1: Convergent index $E_{i}$ with different retained modes.

\begin{tabular}{lcccccccccc}
\hline Group & 1 & 2 & 3 & 4 & 5 & 6 & 7 & 8 & 9 \\
\hline Retained modes & & & & & & & & & & \\
$\quad a$ & 6 & 8 & 10 & 12 & 14 & 16 & 18 & 20 & 22 & 24 \\
$\quad b$ & 12 & 14 & 16 & 18 & 20 & 22 & 24 & 26 & 28 & 30 \\
\hline$C$ & 26859 & 24728 & 24514 & 23767 & 23447 & 23285 & 23182 & 23060 & 22952 & 22711 \\
$E\left(\times 10^{-3}\right)$ & 86.2 & 53.1 & 31.4 & 13.6 & 6.9 & 4.4 & 5.3 & 4.7 & 10.6 & 3.7 \\
\hline
\end{tabular}

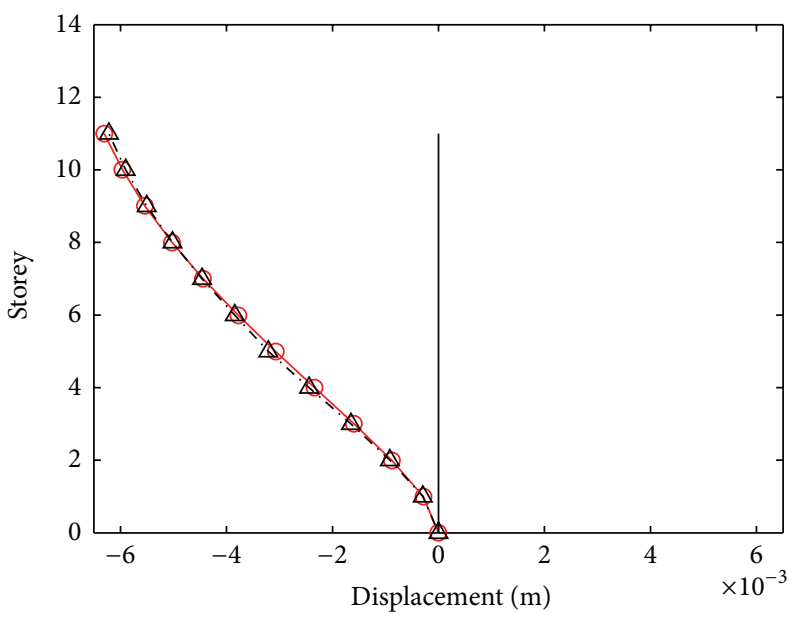

(a)

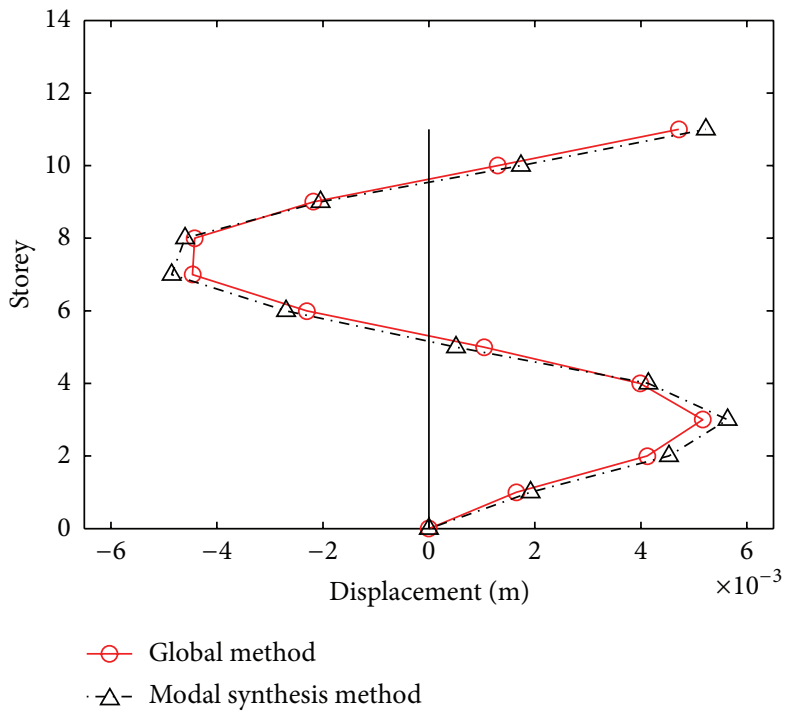

(c)

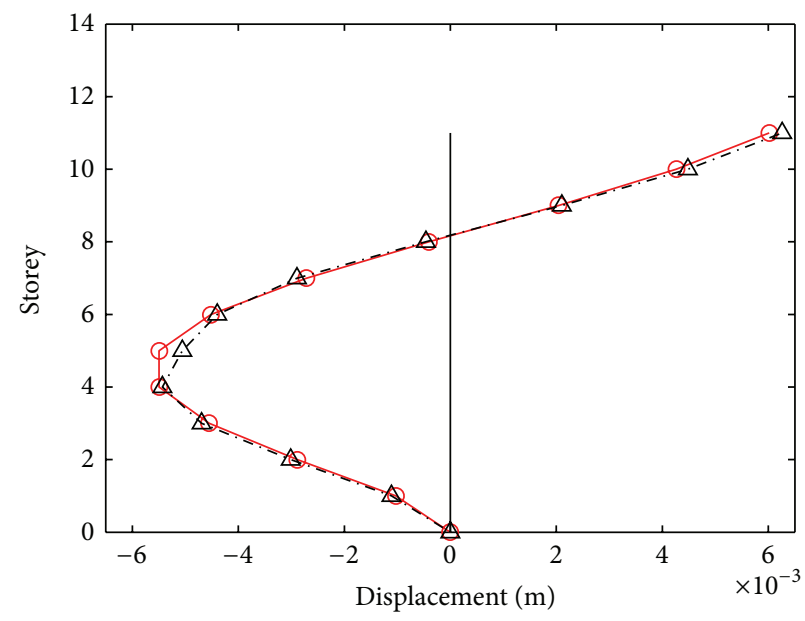

(b)

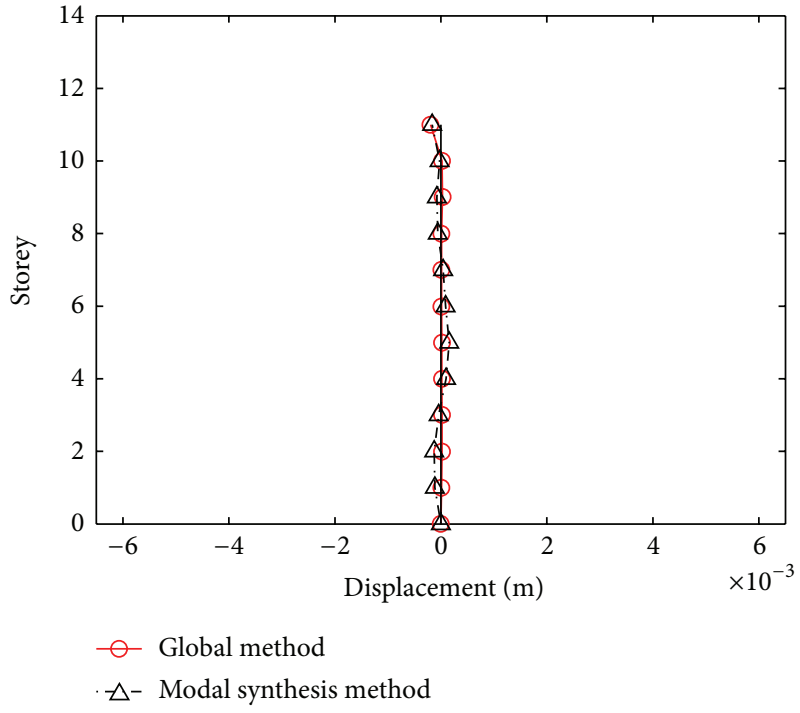

(d)

Figure 3: The first 4 mode shapes after modal synthesis (Group 4).

by how much percentage the sum of eigenvalues will change with the increasing retained modes:

$$
\begin{gathered}
E_{i}=\left|\frac{C_{i+1}-C_{i}}{C_{i+1}}\right| \\
C_{i}=\sum_{k=1}^{n} \omega_{k}^{2} .
\end{gathered}
$$

$C_{i}$ is the sum of eigenvalues after modal synthesis at the $i$ th calculation and $n$ is the first $n$ modes to have certain accuracy.

The convergence index $E_{i}$ with respect to the retained modes of the substructures is listed in Table 1 , the reduction of $E_{i}$ can be observed from Figure 2 clearly, and there is less convergence after Group 4 where 12 and 18 retained modes were included for substructures $a$ and $b$. Table 2 further investigates the modal synthesis method 


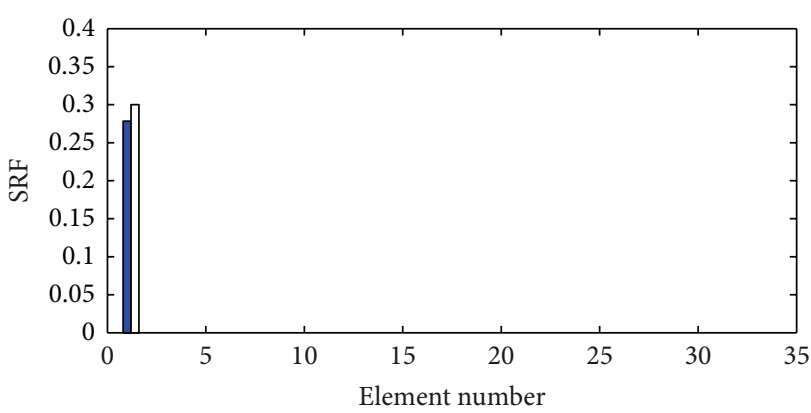

(a) Case 1

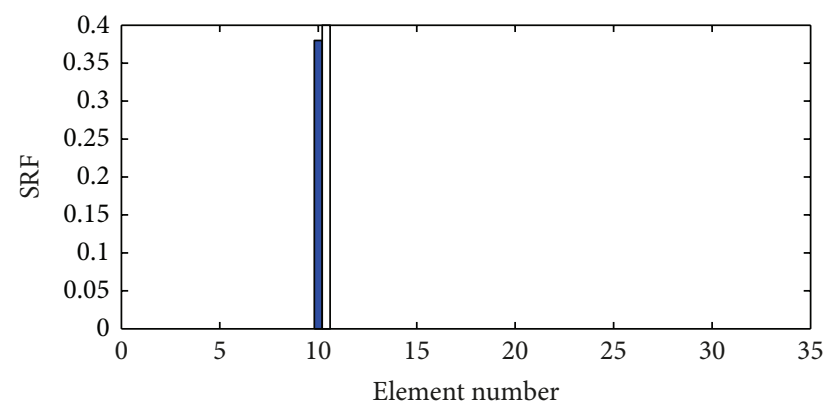

(b) Case 2

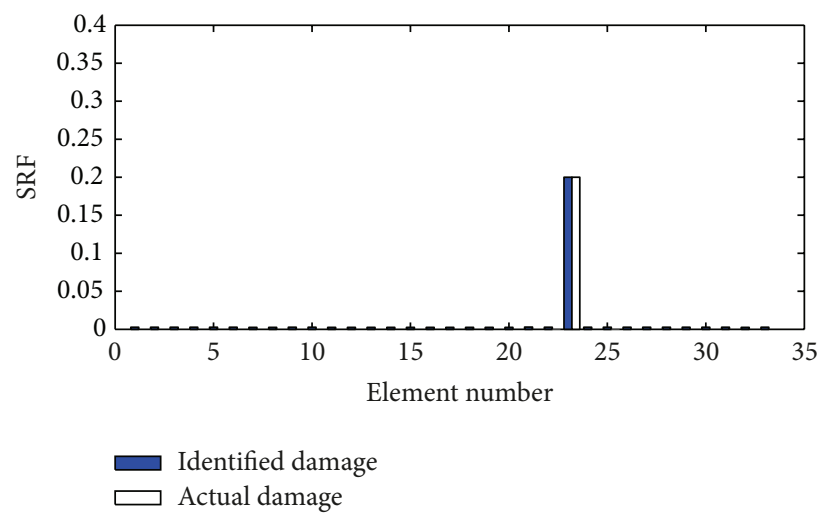

(c) Case 3

FIGURE 4: Damage detection results using free-interface modal synthesis based substructure sensitivity method.

accuracy of Group 4. It is obvious from the table that the differences of both frequencies and MAC are rather small. The first 8 mode shapes after modal synthesis of Group 4 are plotted in Figure 3.

4.2. Substructure Damage Detection on a Frame Structure. Usually, the experimental data collected by applying damages on some elements and the differences between the analytical and the experimental responses are calculated in target function and are to be minimized during the iteration procedure. For the example discussed in this paper, only the first 8 frequencies and mode shapes are included in the target function expressed as

$$
\text { To minimize } \sum_{i=1}^{n}\left(w_{i}\left|f_{i}^{e}-f_{i}^{a}\right|+v_{i}\left|\Phi_{i}^{e}-\boldsymbol{\Phi}_{i}^{a}\right|\right) \text {, }
$$

where the superscript " $e$ " represents the true value, which means value from the damaged model, and " $a$ " represents the analytical value. $w_{i}$ and $v_{i}$ are the weight factors applied to the $i$ th frequency and eigenvector terms, respectively.

The whole structure is simply divided as 2 substructures. Meanwhile, the frequencies and mode shapes of the damaged model are first calculated from finite element model and assumed to be known as experimental data. And all the damages are assumed to be loss in the young's modulus of
TABLE 2: Eigen errors on Group 4.

\begin{tabular}{lcccc}
\hline Modes & $\begin{array}{c}\text { Expected } \\
\text { frequency } \\
(\mathrm{Hz})\end{array}$ & $\begin{array}{c}\text { Analytical } \\
\text { frequency } \\
(\mathrm{Hz})\end{array}$ & $\begin{array}{c}\text { Differences } \\
(\%)\end{array}$ & $\begin{array}{c}\text { MAC } \\
(\%)\end{array}$ \\
\hline 1 & 1.035 & 1.052 & 1.6 & 100 \\
2 & 3.361 & 3.44 & 2.4 & 99.4 \\
3 & 4.359 & 4.512 & 3.5 & 99.5 \\
4 & 6.349 & 6.477 & 2 & 96.4 \\
5 & 7.057 & 7.356 & 4.2 & 95.9 \\
6 & 10.147 & 10.546 & 3.9 & 95.5 \\
7 & 11.536 & 11.719 & 1.6 & 97.6 \\
8 & 14.253 & 14.709 & 3.2 & 97.1 \\
\hline
\end{tabular}

some elements, and their damage extents are defined by SRF values:

$$
\mathrm{SRF}_{i}=\frac{\Delta E_{i}}{E_{i}}=\frac{E_{i}^{u}-E_{i}^{o}}{E_{i}^{u}}
$$

Three single damage cases are first studied, Case 1, Case 2, and Case 3; assumed elemental stiffness reduction happens on elements 1,10 , and 23 referring to Figure 1 by $30 \%, 40 \%$, and $20 \%$, respectively, while other elements remain unchanged. Figure 4 shows the damage detection results by the proposed free-interface modal synthesis based sensitivity method for the three cases, from which one can see that the proposed 


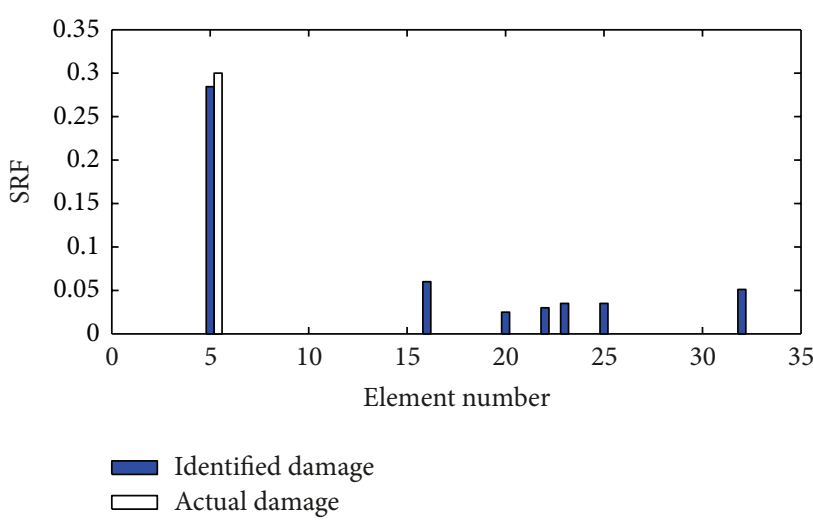

(a) Substructure sensitivity method

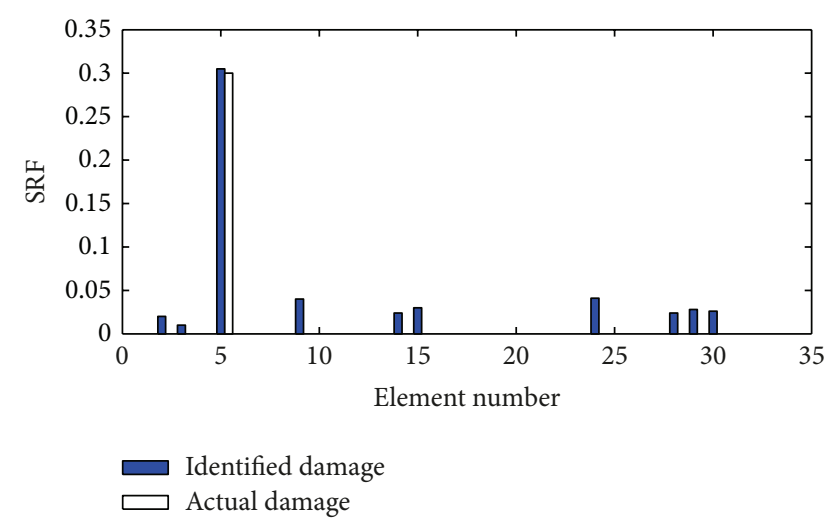

(b) Global sensitivity method

FIGURE 5: Damage detection results (Case 4).

method can detect the damage location perfectly. And the identified damage extents are very close to the true value. Noticing that, although in Case 1 several false detections are found in those undamaged elements, the maximum SRF for these false detection elements is below 0.05, which is ignorable compared to the real damage. It is believed that by optimal choosing the target function for different damage scenario can improve the damage identification results. Therefore, the feasibility of the proposed method can be verified.

\subsection{Damage Detection Ability Compared with Traditional} Sensitivity Method. In this session, in order to evaluate the detection effectiveness of the proposed method, more damage cases are chosen randomly, including single damage and multidamage cases. The damage detection results of the proposed substructure method are compared with those by traditional global sensitivity method. Three damage cases are listed in Table 3 , in which Case 4 is a single damage case, while Cases 5 and 6 are multidamage cases.

For Case 4, both the global and the proposed substructure sensitivity method can detect the damage location and damage extent as well. As compared in Figure 5, for this damage scenario, the traditional sensitivity method will have better detection results. The proposed substructure method has several elements being falsely detected, and some of the SRF values are approximate to 0.1 , which is larger than that of the global sensitivity method with maximum false detection value being 0.035 . However, the comparison of the updated frequencies and mode shapes with true values listed in Table 4 shows little differences in both first ten frequencies and mode shapes, which proves the feasibility of the proposed method in model updating.

Further examining the convergent procedure, the SRF values of the proposed substructure on iterations $1,3,4$, and 5 are graphed in Figure 6, from which one can see that the proposed method is able to locate the damage in the very beginning. And along with the convergence, approximate damage extent was found on iteration 4 . As compared to the
TABLE 3: Damaged Cases 4, 5, and 6.

\begin{tabular}{lcc}
\hline & Damage location & SRF \\
\hline Case 4 & Element 5 & $-30 \%$ \\
Case 5 & Element 3 and 8 & $-30 \%$ and $-20 \%$ \\
Case 6 & Element 1, 2, and 7 & $-30 \%,-50 \%$, and $-20 \%$ \\
\hline
\end{tabular}

TABLE 4: Frequencies and mode shapes using the proposed substructure method.

\begin{tabular}{lcccc}
\hline Mode & $\begin{array}{c}\text { Experimental } \\
\text { data (Hz) }\end{array}$ & $\begin{array}{c}\text { Frequency } \\
\text { Analytical } \\
\text { data (Hz) }\end{array}$ & $\begin{array}{c}\text { Difference } \\
(\%)\end{array}$ & MAC (\%) \\
\hline 1 & 1.032 & 1.033 & 0.1 & 100 \\
2 & 3.353 & 3.353 & 0 & 100 \\
3 & 4.29 & 4.291 & 0.02 & 100 \\
4 & 6.32 & 6.321 & 0.02 & 100 \\
5 & 6.967 & 6.969 & 0.03 & 100 \\
6 & 10.129 & 10.137 & 0.08 & 99.9 \\
7 & 11.46 & 11.462 & 0.02 & 100 \\
8 & 14.155 & 14.162 & 0.05 & 99.8 \\
9 & 14.283 & 14.292 & 0.06 & 99.8 \\
10 & 16.65 & 16.653 & 0.02 & 99.7 \\
\hline
\end{tabular}

global method in Figure 7, where SRF values of iterations 1, 4, 9 , and 10 are graphed, the damage location has not been found until iteration 10. As it comes to the damage extent referred to the convergent curves in Figure 8, the global sensitivity method will have a better convergent and a faster convergent rate in this case.

Case 5 is a multidamage case with two elements assumed to have stiffness reduction of $30 \%$ and $20 \%$, respectively. Figure 9 compares the detection results of the two methods. Along with some elements being falsely identified with the maximum SRF less than 0.05 , the proposed substructure method was able to identify the damage location. And the identified SRF values of the damaged elements are $28.0 \%$ 


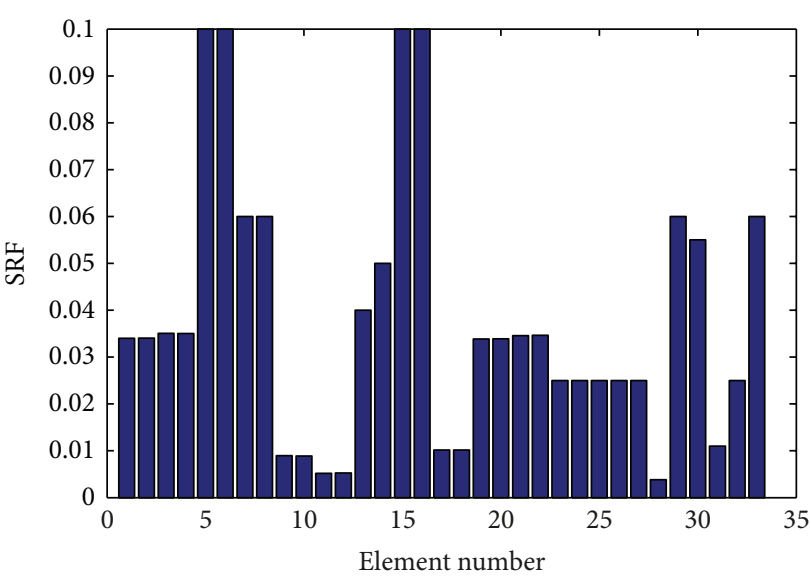

(a)

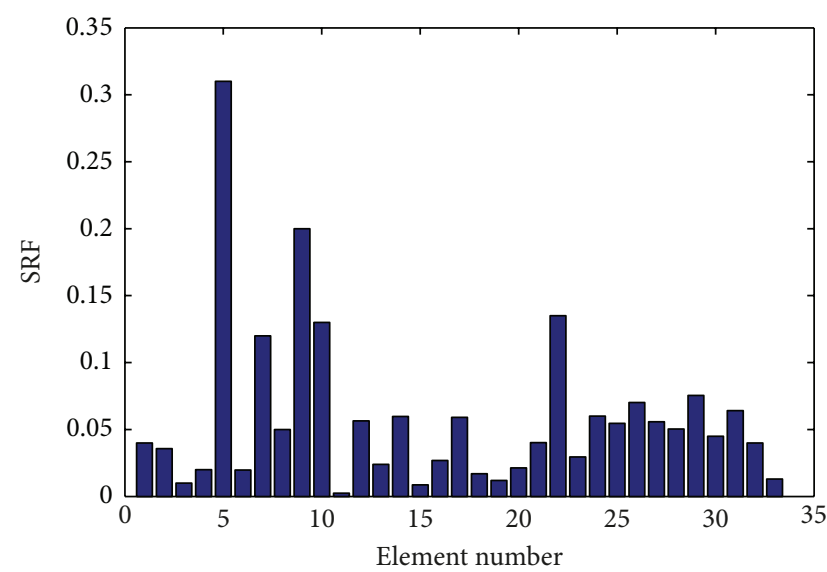

(c)

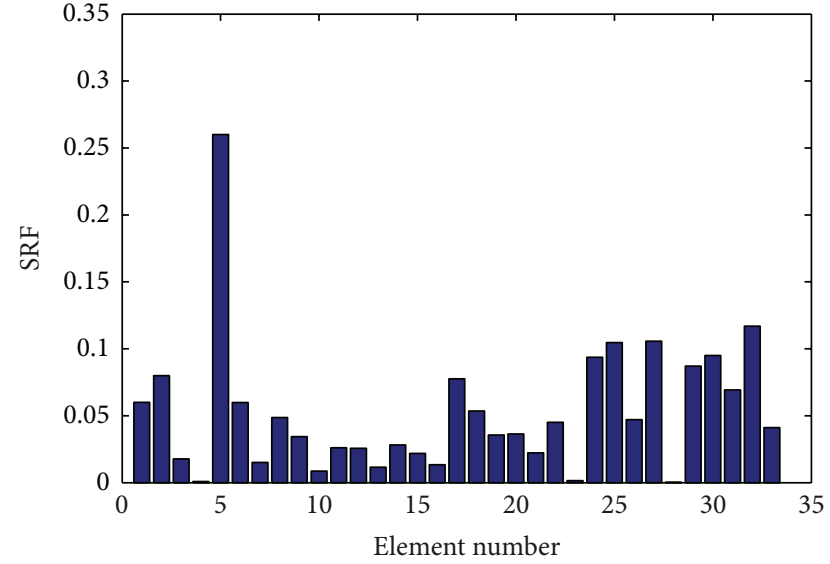

(b)

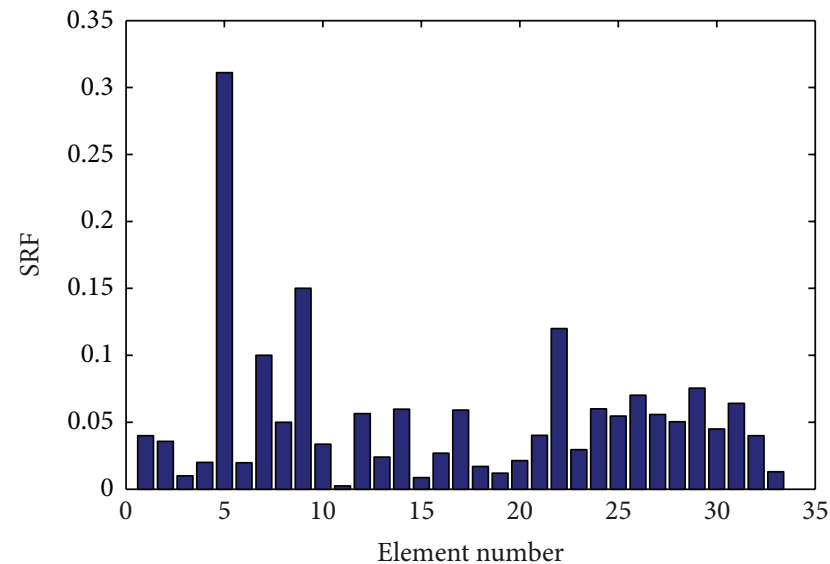

(d)

FIGURE 6: SRF values of substructure sensitivity method (iterations 1, 3, 4, and 5).

and $20.3 \%$, which are very close to the true values. As for the global sensitivity method in this case, it fails to identify the damaged elements. Instead of elements 3 and 8 , the global method has elements 4 and 7 to be the damaged elements. It is observed from the convergent curves (Figure 10) that the substructure sensitivity method gives a relatively smooth fluctuant and overall steady reduction convergent curve, while the global method gives a convergent curve with severe turbulence in this case. And it refuses to converge only after the value of the target function reaches to 3.3.

Figure 11 shows the identified results of Case 6 where 3 elements are assumed to have stiffness reduction by $30 \%$, $50 \%$, and $20 \%$. The proposed substructure method identifies exact damage locations, and the identical SRF values for elements 1,2 , and 7 are $27.2 \%, 47.0 \%$, and $18.1 \%$, which are very similar to the preset values $30 \%, 50 \%$, and $20 \%$. Still there are some falsely identified values in some undamaged elements, but all these values are below 0.05 . As for the traditional global method, it also detects the damage location correctly, and the identified SRF values are $33.1 \%, 49.1 \%$, and $19.8 \%$, which are also approached to the true value. However, compared to the substructure results, the false identified problem is more serious. For example, the identified SRF value of element 14 is $11.9 \%$.

\section{Concluding Remarks}

A free-interface modal synthesis method is applied to civil structures, and, by introducing to the sensitivity method, a free-interface modal synthesis based substructure sensitivity method is proposed for damage detection. The derivatives of eigenvalue and eigenvector with respect to elemental parameters are calculated with substructure approach and herein facilitate the calculation burden of sensitivity matrix.

The application of modal synthesis method and the effectiveness of the proposed substructure damage detection method were verified through a frame structure. As the mode truncation may bring errors to the identification procedure, a criterion for retaining mode numbers of substructure was first proposed. Although there is still some slight false identification, overall, the proposed substructure method was 


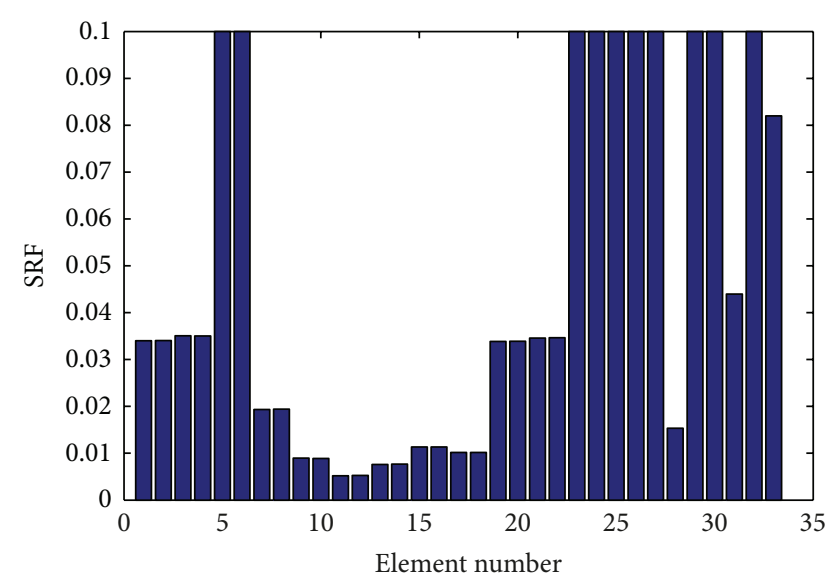

(a)

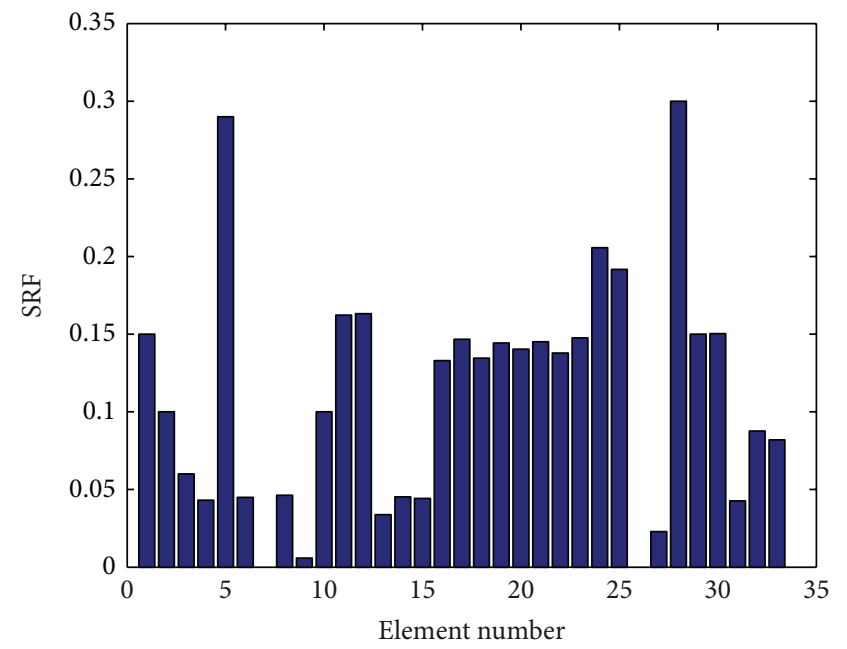

(c)

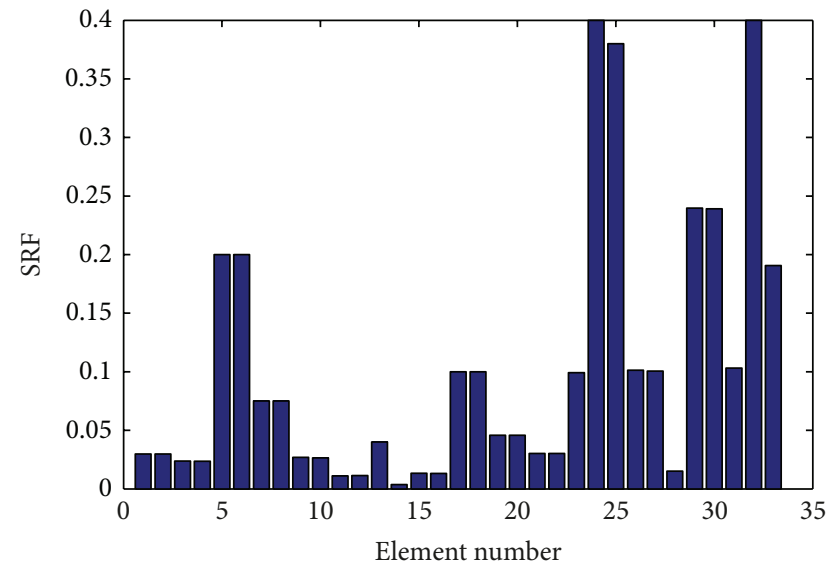

(b)

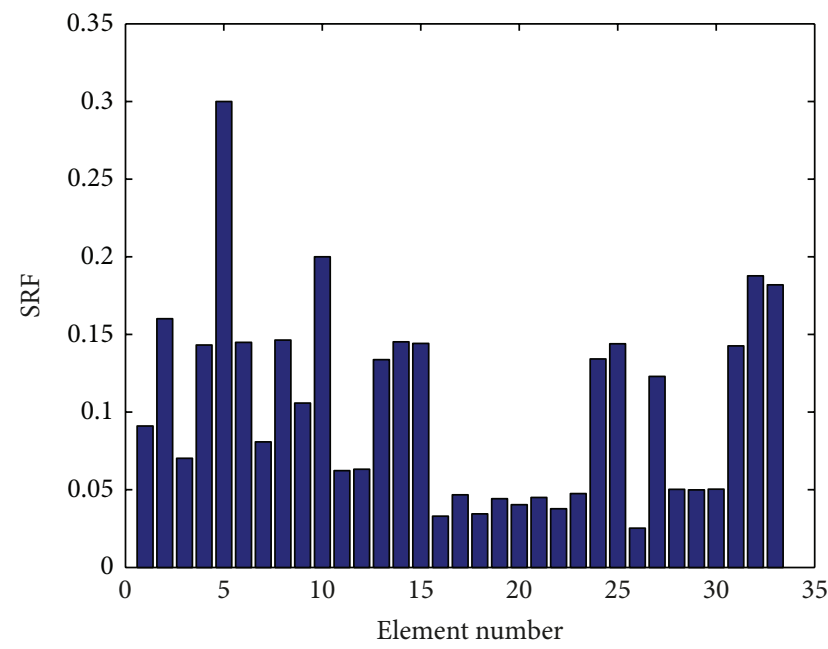

(d)

FIGURE 7: SRF values of global sensitivity method (iterations 1, 4, 9, and 10).

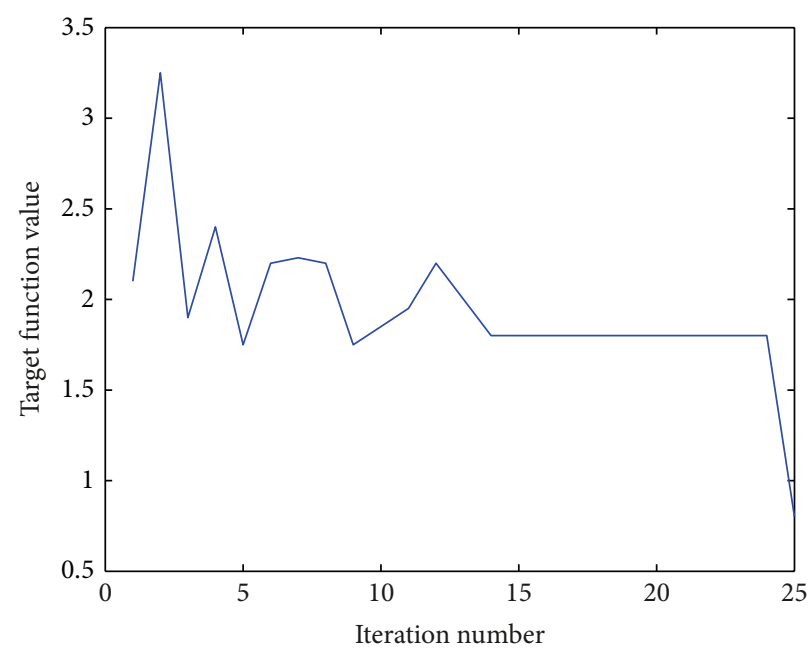

(a) Substructure method

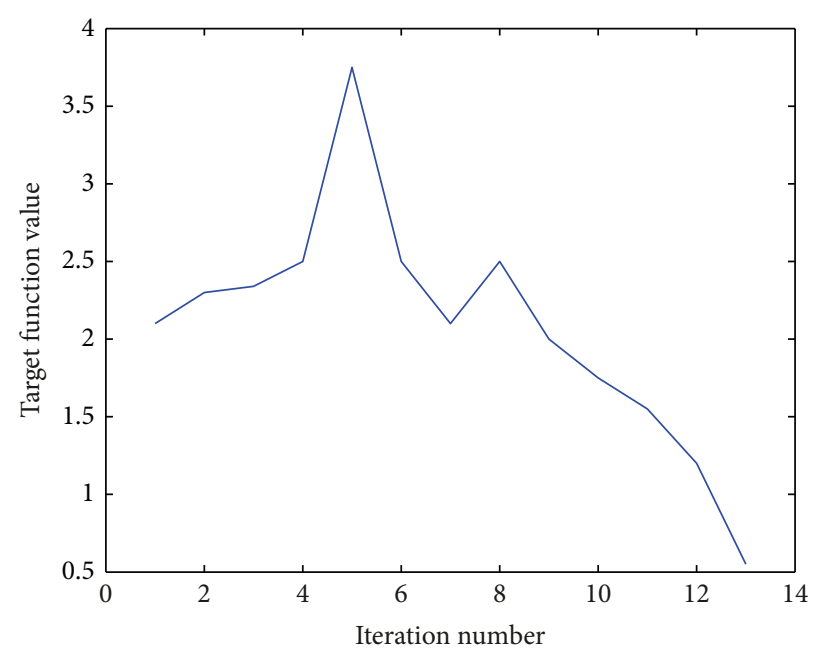

(b) Global method

Figure 8: Convergent curves of Case 4. 


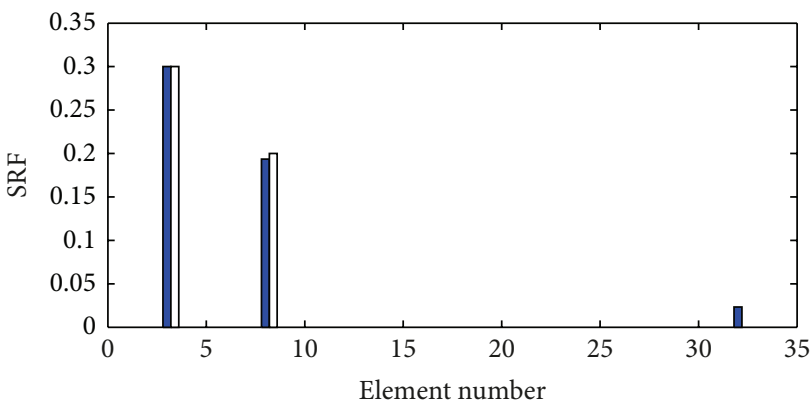

$\square$ Identified damage $\square$ Actual damage

(a) Substructure sensitivity method

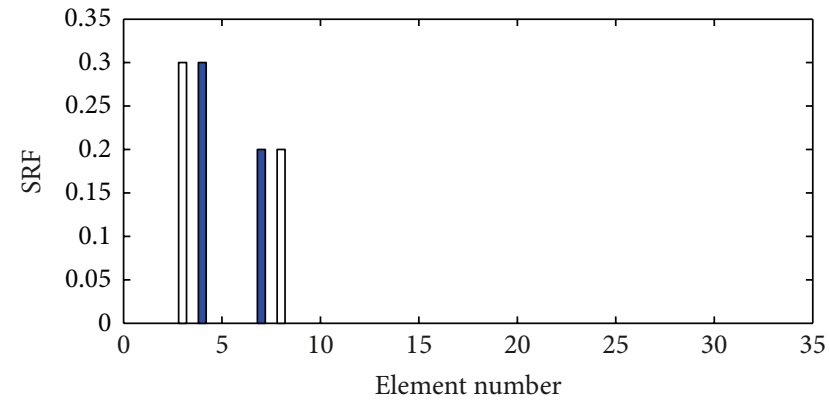

Identified damage Actual damage

(b) Global sensitivity method

Figure 9: Damage detection results (Case 5).

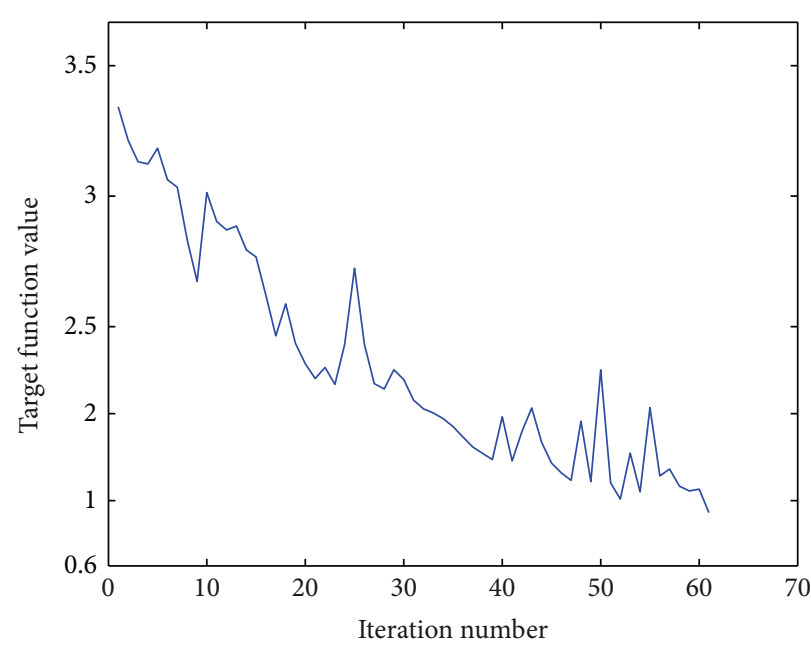

(a) Substructure method

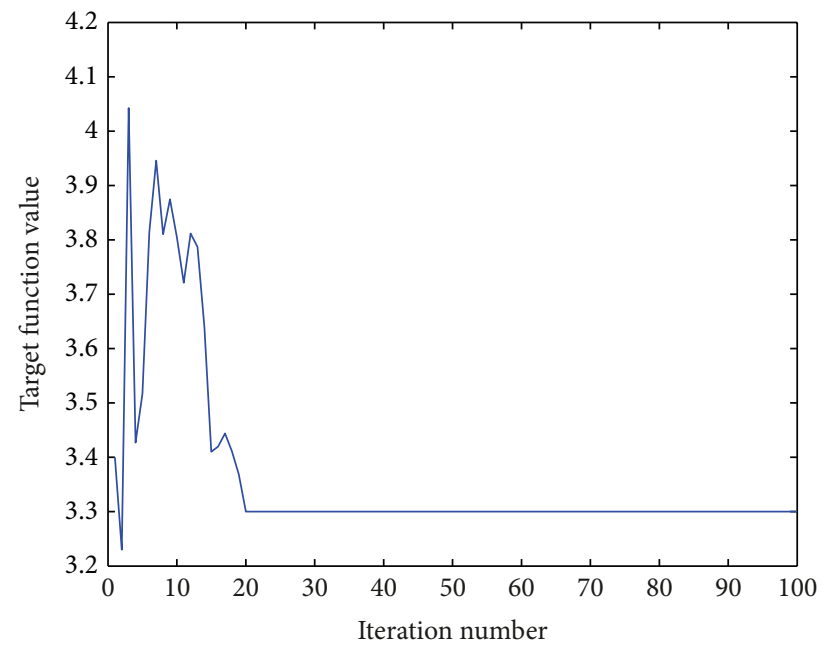

(b) Global method

Figure 10: Convergent curves of Case 5.

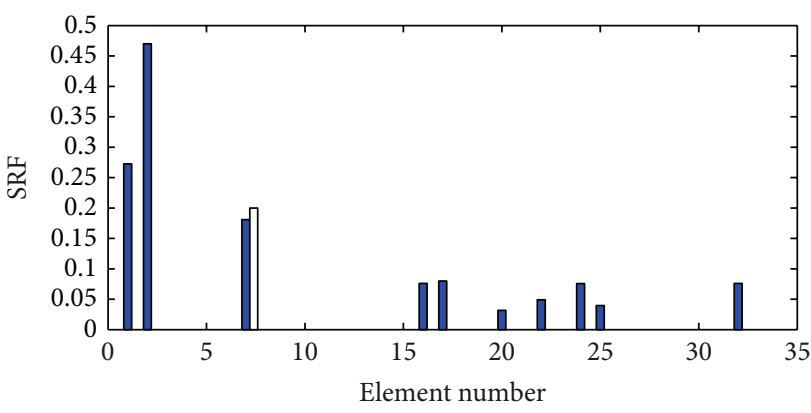

Identified damage Actual damage

(a) Substructure sensitivity method

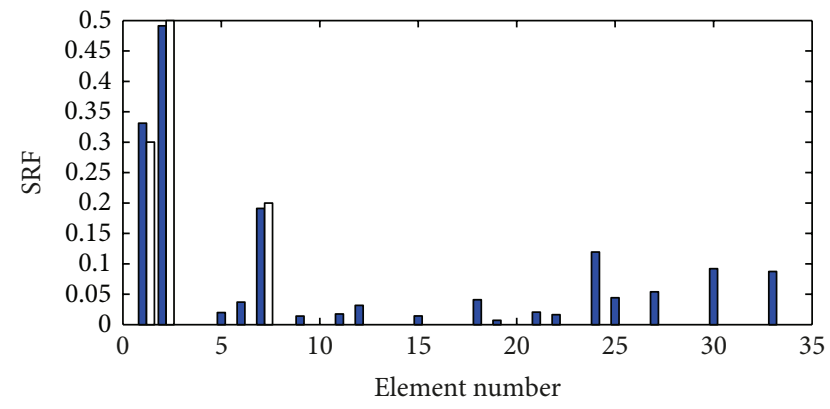

Identified damage Actual damage

(b) Global sensitivity method

FIgURE 11: Damage detection results (Case 6). 
able to identify the damage location as well as the damage extent correctly in both single- and multidamage scenarios. Compared to traditional global sensitivity method, the proposed substructure method has a better speed in detecting the damage location and is even more stable in multidamage cases. More importantly, different from global sensitivity method which takes the whole structure in calculating the sensitivity matrix in every step, the proposed substructure method will only need to calculate and update sensitivity matrix in certain substructure which contains the parameter. And the updating procedure is done within a relative small dimension. By doing so, the proposed substructure method will have fewer calculation and storage burden, and it will be an advantage on model updating efficiency.

\section{Conflict of Interests}

The authors declare that there is no conflict of interests regarding the publication of this paper.

\section{Acknowledgments}

The authors are grateful for financial support by National Natural Science Foundation of China (no. 51108089), Ph.D. Programs Foundation of Ministry of Education of China (no. 20113514120005), and National Natural Science Foundation of Fujian Province (no. 2011J05128).

\section{References}

[1] Y. Xia, Y. Ni, P. Zhang, W. Liao, and J. Ko, "Stress development of a supertall structure during construction: Field monitoring and numerical analysis," Computer-Aided Civil and Infrastructure Engineering, vol. 26, no. 7, pp. 542-559, 2011.

[2] W. H. Chen, Z. R. Lu, W. Lin et al., "Theoretical and experimental modal analysis of the Guangzhou New TV Tower," Engineering Structures, vol. 33, no. 12, pp. 3628-3646, 2011.

[3] Y. Q. Ni, Y. Xia, W. Lin, W. H. Chen, and J. M. Ko, "SHM benchmark for high-rise structures: a reduced-order finite element model and field measurement data," Smart Structures and Systems, vol. 10, no. 4, pp. 411-426, 2012.

[4] B. Chen, Y. L. Xu, and X. Zhao, "Integrated vibration control and health monitoring of building structures: a time-domain approach," Smart Structures and Systems, vol. 6, no. 7, pp. 811833,2010

[5] B. Chen, S. Zhao, and P. Li, "Application of Hilbert-HUAng Transform in Structural HEAlth Monitoring: A STAte-of-theART Review," Mathematical Problems in Engineering, Article ID 317954, ART. ID , 22 pages, 2014.

[6] C. B. Yun, J. J. Lee, and K. Y. Koo, "Smart structure technologies for civil infrastructures in Korea: recent research and applications," Structure and Infrastructure Engineering, vol. 7, no. 9, pp. 673-688, 2011.

[7] Y. Xia, Y. Ni, P. Zhang, W. Liao, and J. Ko, "Stress development of a supertall structure during construction: field monitoring and numerical analysis," Computer-Aided Civil and Infrastructure Engineering, vol. 26, no. 7, pp. 542-559, 2011.
[8] Y. Fujino, D. M. Siringoringo, and M. Abe, "The needs for advanced sensor technologies in risk assessment of civil infrastructures," Smart Structures and Systems, vol. 5, no. 2, pp. 173191, 2009.

[9] J. M. Ko and Y. Q. Ni, "Technology developments in structural health monitoring of large-scale bridges," Engineering Structures, vol. 27, no. 12, pp. 1715-1725, 2005.

[10] H. Li, T. Yi, M. Gu, and L. Huo, "Evaluation of earthquakeinduced structural damages by wavelet transform," Progress in Natural Science, vol. 19, no. 4, pp. 461-470, 2009.

[11] T. H. Yi, H. N. Li, and H. M. Sun, "Multi-stage structural damage diagnosis method based on "energy-damage" theory," Smart Structures and Systems, vol. 12, no. 3-4, pp. 345-361, 2013.

[12] B. Chen, Z. W. Chen, G. J. Wang, and P. X. Wei, "Damage detection on sudden stiffness reduction based on discrete wavelet transform," The Scientific World Journal, vol. 2014, Article ID 807620, 16 pages, 2014.

[13] B. Jaishi and W. X. Ren, "Structural finite element model updating using ambient vibration test results," Journal of Structural Engineering, vol. 131, no. 4, pp. 617-628, 2005.

[14] T. Yi, H. Li, and M. Gu, "Optimal sensor placement for structural health monitoring based on multiple optimization strategies," Structural Design of Tall and Special Buildings, vol. 20, no. 7, pp. 881-900, 2011.

[15] Y. Xia, H. Hao, A. J. Deeks, and X. Zhu, "Condition assessment of shear connectors in slab-girder bridges via vibration measurements," Journal of Bridge Engineering, vol. 13, no. 1, pp. 43$54,2008$.

[16] E. Görl and M. Link, "Damage identification using changes of eigenfrequencies and mode shapes," Mechanical Systems and Signal Processing, vol. 17, no. 1, pp. 103-110, 2003.

[17] Z. R. Lu and S. S. Law, "Features of dynamic response sensitivity and its application in damage detection," Journal of Sound and Vibration, vol. 303, no. 1-2, pp. 305-329, 2007.

[18] S. S. Law, K. Zhang, and Z. D. Duan, "Structural damage detection from coupling forces between substructures under support excitation," Engineering Structures, vol. 32, no. 8, pp. 2221-2228, 2010.

[19] S. S. Law and J. Li, "Updating the reliability of a concrete bridge structure based on condition assessment with uncertainties," Engineering Structures, vol. 32, no. 1, pp. 286-296, 2010.

[20] H. Chen and N. Bicanic, "Assessment of damage in continuum structures based on incomplete modal information," Computers and Structures, vol. 74, no. 5, pp. 559-570, 2000.

[21] S. Živanović, A. Pavic, and P. Reynolds, "Finite element modelling and updating of a lively footbridge: the complete process," Journal of Sound and Vibration, vol. 301, no. 1-2, pp. 126-145, 2007.

[22] S. Weng, Y. Xia, Y. Xu, X. Zhou, and H. Zhu, "Improved substructuring method for eigensolutions of large-scale structures," Journal of Sound and Vibration, vol. 323, no. 3-5, pp. 718-736, 2009.

[23] G. Kron, Diakoptics: The Piecewise Solution of Large-Scale Systems, Macdonald and Co., London, UK, 1963.

[24] J. Li, S. S. Law, and H. Hao, "Improved damage identification in bridge structures subject to moving loads: numerical and experimental studies," International Journal of Mechanical Sciences, vol. 74, pp. 99-111, 2013.

[25] J. Li, S. S. Law, and Y. Ding, "Substructure damage identification based on response reconstruction in frequency domain and model updating," Engineering Structures, vol. 41, pp. 270-284, 2012. 
[26] J. Li and S. S. Law, "Substructural damage detection with incomplete information of the structure," Journal of Applied Mechanics, vol. 79, no. 4, Article ID 041003, 2012.

[27] J. Li and S. S. Law, "Substructural response reconstruction in wavelet domain," Journal of Applied Mechanics, vol. 78, no. 4, Article ID 041010, 10 pages, 2011.

[28] S. Weng, Y. Xia, Y. L. Xu, and H. P. Zhu, "Substructure based approach to finite element model updating," Computers and Structures, vol. 89, no. 9-10, pp. 772-782, 2011.

[29] S. Weng, Y. Xia, X. Zhou, Y. Xu, and H. Zhu, "Inverse substructure method for model updating of structures," Journal of Sound and Vibration, vol. 331, no. 25, pp. 5449-5468, 2012. 

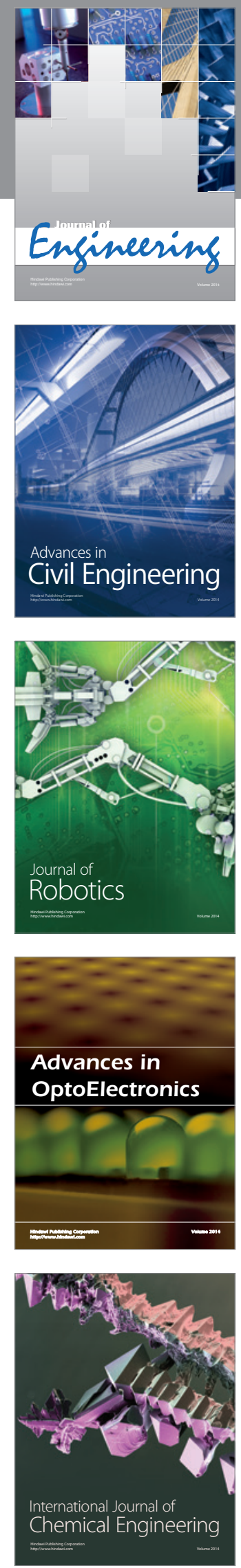

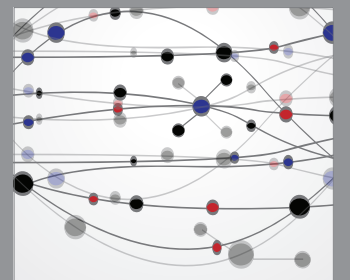

The Scientific World Journal
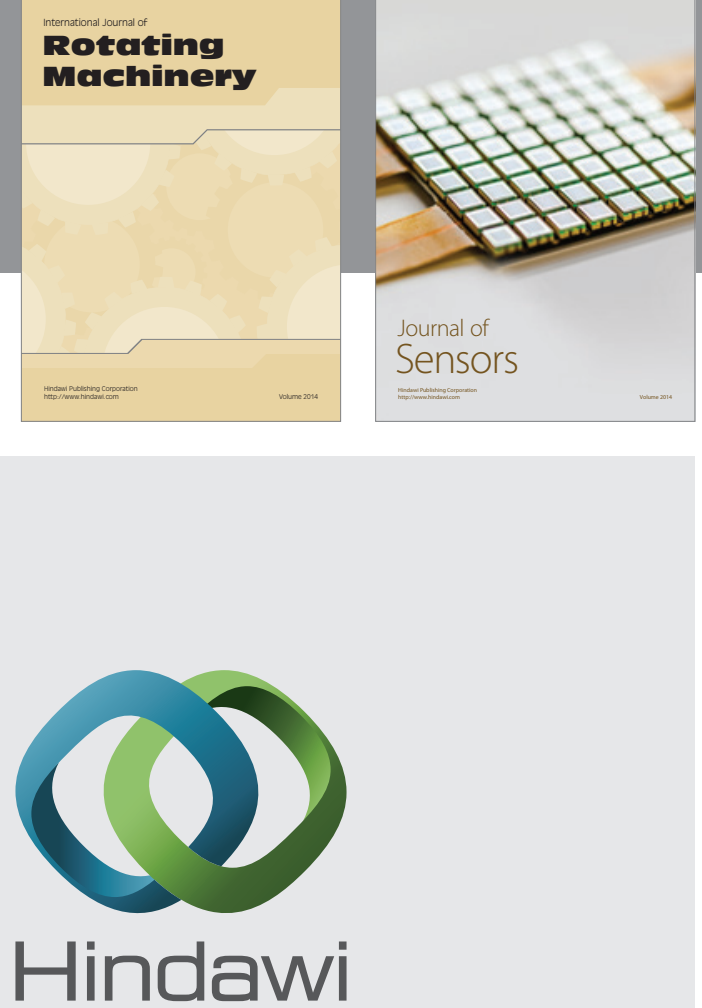

Submit your manuscripts at http://www.hindawi.com
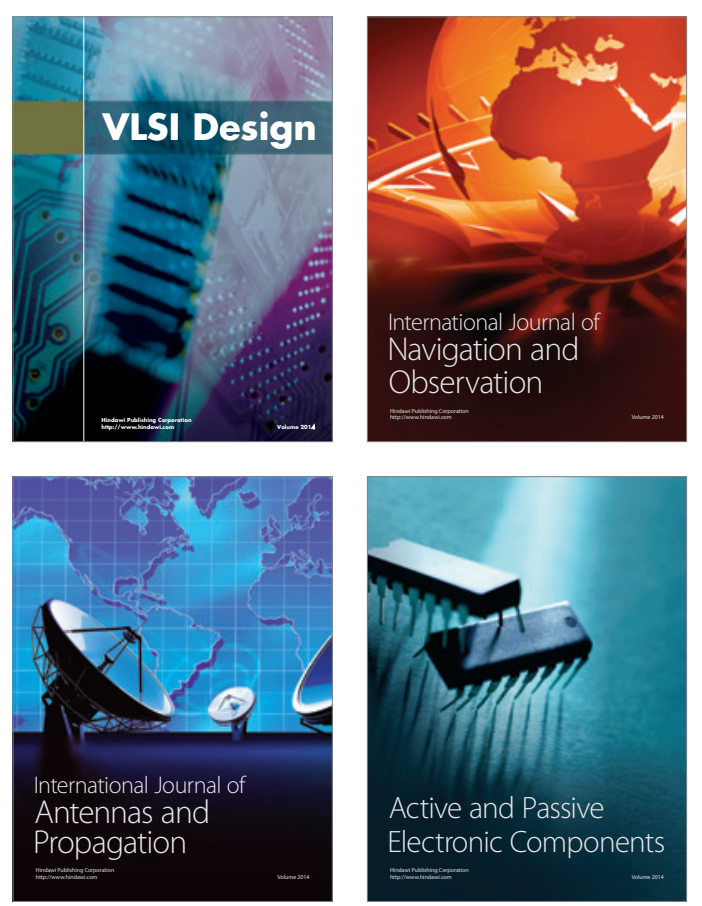
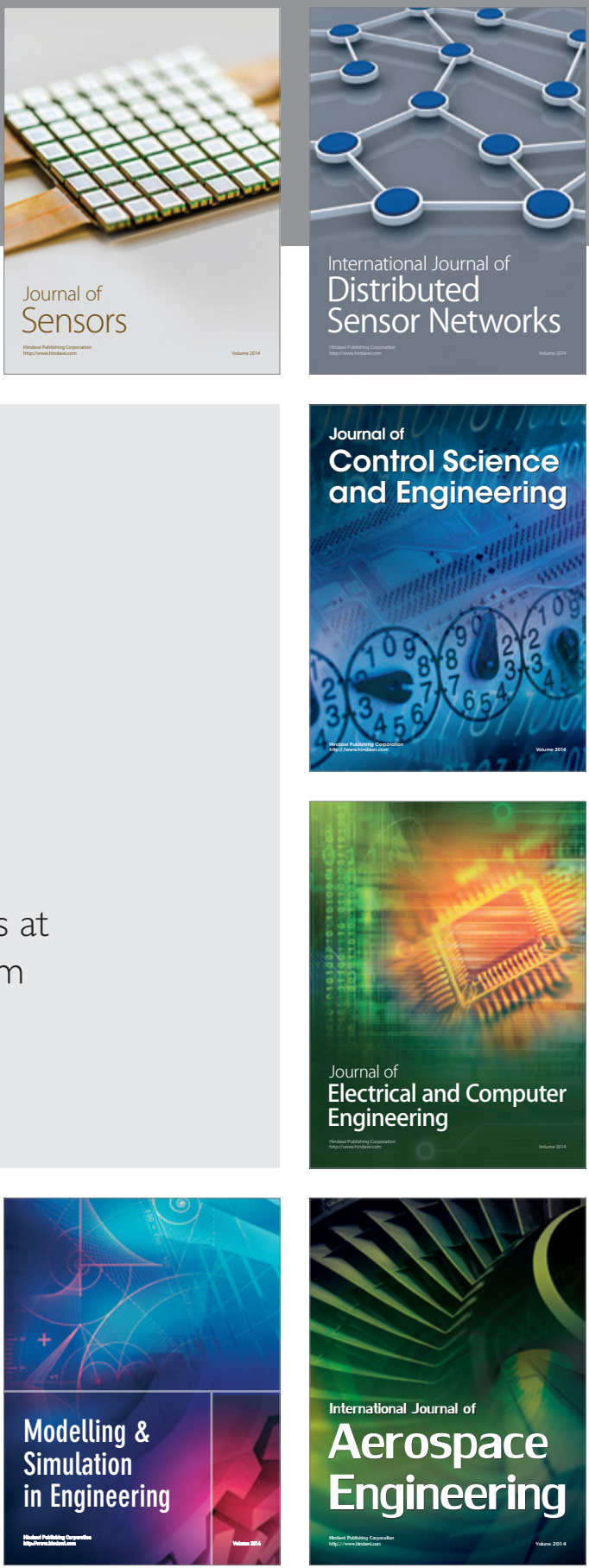

Journal of

Control Science

and Engineering
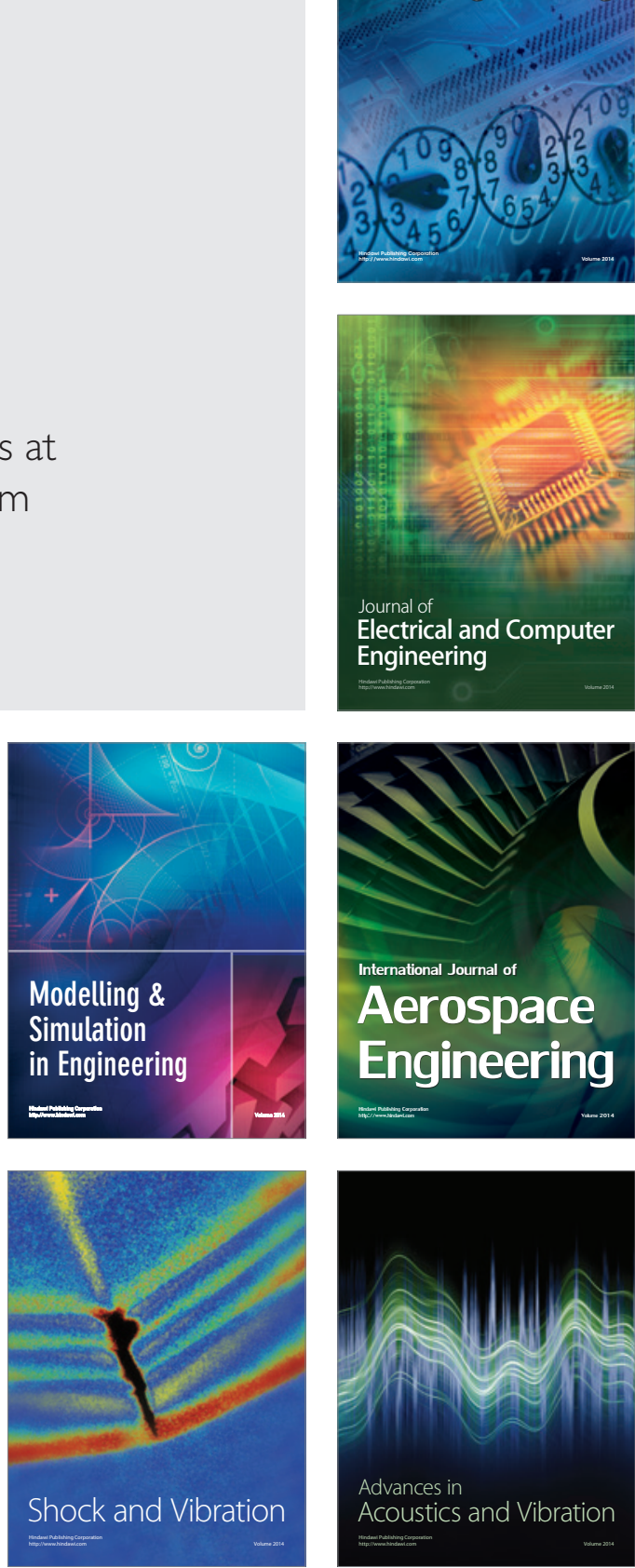